\title{
25 Research Suare \\ The Study on Angiotensin II Induced-ferroptosis in Vascular Endothelial Cells
}

\section{Hong Fang}

Tongji University

Chi Liu

Jing'an District Centre Hospital of Shanghai

Omer Cavdar

Tongji University

Yi Shen ( $\sim$ liling196801@126.com )

Tongji Hospital, School of Medicine, Tongji University

\section{Original investigation}

Keywords: Angiotensin II, ferroptosis, vascular endothelial cells

Posted Date: June 7th, 2021

DOl: https://doi.org/10.21203/rs.3.rs-506444/v1

License: (c) (i) This work is licensed under a Creative Commons Attribution 4.0 International License.

Read Full License 


\section{Abstract}

Background: To verify the effect of Angiotensin II on ferroptosis in vascular endothelial cells and clarify the related mechanism.

Methods: HUVECs were evaluated for p53, P21, ALOX12, VEGF, MDA, GSH. Molecular marker impact upon Angll-induced ferroptosis was evaluated with students' t-test囚one-way analysis of variance (ANOVA).

Results: As the concentration of Ang II increased冈the level of ALOX12, P53, GSH and MDA increased in HUVECs. The expression of VEGFA in HUVECs is negatively correlated with dose of Ang II. Incubation of HUVECs in Angll and valsartan for 48hr reduces ALOX12, P21, GSH and MDA. Compared with the single Angll group, ALOX12, P21, GSH and MDA in valsartan group was decreased significantly $₫ p=0.000 \rrbracket$. In pifithrin-a hydrobromide-treated, ALOX12, P21, GSH and MDA was reduced significantly, as compared to

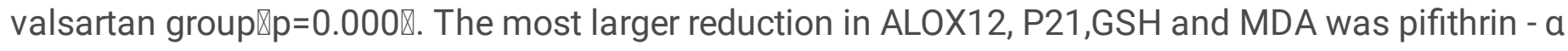
hydrobromide combined with valsartan group. In contrast, the expression of VEGFA increased significantly after HUVECs were treated with pifithrin - $a$ hydrobromide and valsartan $\unrhd p=0.000 \rrbracket$.

Conclusions: Angll can induce ferroptosis of vascular endothelial cells in a dose-dependent manner. The mechanism of Angll-induced ferroptosis may be regulated through the signal axis of ATR1,2-p53-ALOX12.

\section{Background}

Ferroptosis is a new form of programmed cell death that is different from apoptosis,necroptosis and autophagy at both the morphological and biochemical levels and has characteristic accumulation of reactive oxygen species (ROS) resulting from iron accumulation and lipid peroxidation. Recent studies have shown that ferroptosis plays an important regulatory role in the occurrence and development of many diseases and has become the focus and hotspot of research on the treatment and prognosis improvement of related diseases. [1-4]

The endothelium lies in between the circulating blood and vascular smooth muscle cells, which are responsible for peripheral resistance. It may be easily damaged and endothelial dysfunction occurs in the pathogenesis of various cardiovascular complications, particularly in hypertension. Endothelial dysfunction contributes to an increase in large arterial stiffness in patients with isolated systolic hypertension, resulting in impaired vascular elasticity and compliance, and subsequent arterial hypertension. Evidence from previous studies suggest that angiotensin II (AngII), a peptide of the rennin angiotensin system, is considered a proinflammatory mediator in hypertension through mechanisms involving production of ROS. [5, 6]However, it is unclear whether Angll can induce ferroptosis in vascular endothelial cells and the underlying mechanism remains elusive. Therefore, in the present study, we investigated the effect of Angll on vascular endothelial ferroptosis and the possible mechanism using human umbilical vein endothelial cells (HUVECs).

\section{Materials And Methods}




\subsection{Cell culture}

For all cell culture studies, primary HUVECs were used. HUVECs were cultured in $1.5 \%$ gelatin-coated cell culture dishes, and cells at passages 4-8 were used in the experiments. [7] At first, the cells were divided into the following six groups: control group; $0.1 \mathrm{uM}$ Angll ; 1 uM Angll ; 10uM Angll ; 100uM Angll ; 1000uM Angll. Different groups were treated with different concentrations of Angll. In a second experiment, cells in all groups were treated with ang II. Then, cells in four groups were treated in different methods respectfully. Valsartan was used in AT1R blocking group. The p53 block group was incubated with pifithrin-a hydrobromide. AT1R and p53 simultaneously blocked group was treated with valsartan and pifithrin-a hydrobromide at the same time.

\subsection{Measurement of Glutathione and Malonaldehyde}

The concentration of reduced glutathione(GSH) and malonaldehyde(MDA) released by HUVECs was evaluated using an ELISA assay. Briefly, $100 \mu \mathrm{L}$ different dilutions of the standard sample were added to the reaction plate and incubated at $37^{\circ} \mathrm{C}$ for 30 minutes. Secondly, to each well, $100 \mu \mathrm{L}$ of test samples was added to the plate after washes and incubated at $37^{\circ} \mathrm{C}$ for 2 hours. Thereafter, the plates were washed before the addition of $100 \mu \mathrm{L}$ horseradish peroxidase-labeled secondary antibody and incubated at $37^{\circ} \mathrm{C}$ for 30 minutes. Afterward, $50 \mu \mathrm{L}$ developer $\mathrm{A}$ and $50 \mu \mathrm{L}$ developer $\mathrm{B}$ were added. The plates were kept in the dark for 15 minutes. Lastly, to each well, $50 \mu \mathrm{L}$ stop solution was added into the plate to terminate the reaction. The optical density of the plate was read using an ELISA reader, and the concentration of the test sample was calculated.[8]

\subsection{Determination of Arachidonic acid 12-lipoxygenase, Vascular endothelial growth factor, p53, P21}

After treatment with the corresponding drugs, HUVECs were lysed in RIPA buffer containing a mixture of protease inhibitors and centrifuged to extract total protein. Protein concentrations were determined using a BCA protein assay kit according to the manufacturer's instructions, and $25 \mu \mathrm{g}$ of protein was analyzed by $8 \%$ sodium dodecyl sulfate-polyacrylamide gel electrophoresis (SDS-PAGE). Membranes with transferred proteins were blocked with $5 \%$ skim milk for 1 hour and incubated overnight at $4^{\circ} \mathrm{C}$ with antibodies against the following proteins: Arachidonic acid 12-lipoxygenase (ALOX12), Vascular endothelial growth factor (VEGFA),P53,P21, and GAPDH. The relative intensities of protein bands were analyzed using ImageJ software.[9]

\subsection{Statistical analysis}

Mean \pm standard deviation (SD) was displayed to show the data. SPSS 22 statistical software package was used to analyze the data. Students' t-test and one-way analysis of variance (ANOVA) were utilized for the contrast among different groups. Multiple regression assessed associations between ALOX12, P53, P21, VEGFA and vascular endothelial ferroptosis. P values of less than 0.05 were considered statistically significant. 


\section{Results}

\subsection{The relation between concentration of Ang II and vascular endothelial ferroptosis:}

As the concentration of Ang II $(0,0.1,1,10,100$, and $1000 \mathrm{uM}$ for $48 \mathrm{~h})$ increased the level of ALOX12, P53,GSH and MDA increased in HUVECs. The expression of VEGFA in HUVECs is negatively correlated with dose of Ang II. (Fig. 1,2, Table 1,2)

\subsection{Impacts of AT1R antagonist, P53 inhibitor on vascular endothelial ferroptosis}

Incubation of HUVECs in Angll and valsartan for 48hr reduces ALOX12, P21, GSH and MDA. Compared with the single Angll group, ALOX12, P21, GSH and MDA in valsartan group was decreased significantly. In pifithrin-a hydrobromide-treated, ALOX12, P21, GSH and MDA was reduced significantly, as compared to valsartan group. The most larger reduction in ALOX12, P21,GSH and MDA was pifithrin - a hydrobromide combined with valsartan group. In contrast, the expression of VEGFA increased significantly after HUVECs were treated with pifithrin - a hydrobromide and valsartan. (Fig. 2, Table 2)

\section{Discussion}

In the present study, by stimulating HUVECs with angiotensin II, the production of MAD and GSH was significantly promoted. These data indicated that level of MAD and GSH in HUVECs was greatly elevated by angiotensin II, which was a factor that induced HUVEC ferroptosis. The result in our study demonstrated that angiotensin II increased HUVECs ferroptosis in a dose-dependent manner.

Studies have shown that ferroptosis is closely related to the pathophysiological processes of more and more diseases. The discovery of ferroptosis has opened up a new platform in the field of disease research, and its clinical significance in the occurrence, development, and treatment of diseases has gradually emerged. With the deepening of the research, ferroptosis has been found in the pathophysiological processes and diseases, and it provides a new method for treating these diseases.[1$4,10,11]$

Ferroptosis is a form of lipid peroxidation-induced cell death that can be regulated in many ways, from altering the activity of antioxidant enzymes to the level of transcription factors. The p53 is 'the guardian of the genome' that participates in the control of cell survival and division under various stresses. Beyond its effects on apoptosis, autophagy, and cell cycle, p53 also regulates ferroptosis either through a transcriptional or posttranslational mechanism. p53-mediated activation of p21 was reported to promote the conservation of glutathione.[1-4, 10, 11]

ALOX12 is critical for p53-mediated ferroptosis. $[12,13]$ Inactivation of ALOX12 can reduce p53-mediated ferroptosis caused by active oxygen stress. ALOX12 was shown to be related to the ferroptosis independent of ACSL4 [14]. The ALOX12 gene resides on human chromosome 17p13.1.The ALOX12 protein specifically catalyzes the addition of molecular oxygen to arachidonic acid to produce a 
biologically active lipid medium, such as 12-hydroxyeicosatetraenoic acid(12-HETE) [12,13]. As an important lipoxygenase, ALOX12 plays a significant role in biological processes. Its metabolites are not only necessary for normal biological processes but also the basis for many diseases. A large body of evidence indicates that methylation modification of ALOX12 and its genetic variation and oxidative stress in the environment affect the expression and function of ALOX12. Because ALOX12 has the function of regulating platelet aggregation, cell migration, and tumor cell proliferation, it is mainly involved in the development of diseases such as thrombosis, atherosclerosis, and cancer. Besides,ALOX12 is involved in the regulation of inflammation and apoptosis. ALOX12 plays an important role in the occurrence and development of diseases. ALOX12 R261Q is associated with the risk of essential hypertension in the Spanish population. ALOX12 expression was reduced in carotid atherosclerosis. Down-regulation of ALOX12 blocks a response caused by vascular endothelial contraction. In the arteries of mice, ALOX12 metabolizes arachidonic acid to produce thromboxane receptor antagonists, which effectively stretch blood vessels [15]. In addition, the increased expression of ALOX12 in the vascular smooth muscle of hypertensive rats can increase the expression level of Ang II subtype 1 receptor [16], and a study has shown that the increased expression of Ang II receptor is significantly correlated with the severity of pregnancy-induced hypertension [17]. Excess aldosterone can upregulate the expression of ALOX12 and ALOX15 in human vascular smooth muscle, thereby increasing the risk of LDL oxidation and atherosclerosis [18]. In this study, Ang II was used to treat HUVECs; it increased p53 and ALOX12 of HUVECs in dose-dependent manners. The level of MAD, GSH, p53 and ALOX12 in HUVECs was positively correlated to dose of Ang II. Furthermore, our results showed that MAD, GSH and ALOX12 decreased after blocking AT1R and/or p53. ALOX12, P21, GSH and MDA was partially attenuated by the AT1R antagonist. Moreover, the level of ALOX12, P21, GSH and MDA was reduced further in HUVECs treated with both p53 inhibitor and AT1R blocker, indicating the involvement of both AT1R and p53 in the Ang Il-induced HUVECs ferroptosis. A lot of literature reports that AT2R is one of the functional receptors of Angiotensin II, which has been proven to mediate the progress of apoptosis.[8] In our current study, the inhibitory effect of p53 was stronger than that of AT1R, indicating that AT2R may also be involved in this process.

The VEGF family exerts their biological functions through the interaction with transmembrane receptors such as tyrosine kinase receptors VEGFR1 and VEGFR2. The ligands which specifically bind to VEGFR1 are VEGF-A, -B and PIGF while those bind to VEGFR2 are VEGF-A, -C, -D and -E4,5. Binding of VEGFs to VEGF receptor-1 and - 2 triggers downstream signaling pathways resulted in EC proliferation, migration, invasion and high vascular permeability. Pfaff et al. have reported that augmentation of p53 expression could decrease the levels of VEGFA in an ischemia-induced angiogenesis and arteriogenesis mouse model. However, the roles of p53 in the regulation of VEGFA have always been controversial. Quite a few studies have reported that p53 inhibits the expression of VEGFA. Other studies have reported that the expression of p53 is positively related to the expression of VEGFA.[19-24] Chen et al. reported that p53 suppressed expression of VEGFA through miR-1249.[25] The ALOX12 product 12S-HETE also increased the release of VEGF, whereas ALOX12 inhibitors had the opposite effect, suggesting that 12S-HETE is also an endogenous regulator of VEGF secretion by these cells. Overexpression of ALOX12 had a similar effect. [26]These controversial results may be due to different cells. In our study, we have founded that 
VEGF is negatively correlated with dose of Ang II in HUVECs. However, there was a positive correlation between p53 and Angll. The expression of VEGFA increased after AT1R and/or p53 was inhibited. These results indicate that over high concentration of Angll can promote the production of p53, inhibit the expression of VEGFA, and induce ferroptosis of vascular endothelial cells.

Our study is limited by the fact that we did not observe the effect of AT2R and ALOX12 to be blocked in HUVECs directly. Another limitation is that there are few indicators to evaluate ferroptosis in our study. In spite of these limitations, the data from our experiments can still reveal many problems.

\section{Conclusion}

In conclusion, our present results suggest that Angll can induce ferroptosis of vascular endothelial cells in a dose-dependent manner. The mechanism of Angll-induced HUVECs ferroptosis may be regulated through the signal axis of ATR1,2-p53-ALOX12.

\section{Declarations}

\section{Declaration of Competing Interest}

The authors declare that they have no conflict of interest.

\section{Ethics approval and consent to participate}

Not applicable

Consent for publication

Not applicable

\section{Availability of data and materials}

Not applicable

\section{Competing interests}

Not applicable

\section{Funding}

Not applicable

\section{Authors' contributions}

Ph.D. Hong Fang Department of Cardiology, Tongji Hospital, School of Medicine, Tongji University, Shanghai 200065, China ${ }^{1}$ 
M.D. Chi Liu National clinical research center for aging and medicine, Jing an district Central Hospital, Shanghai, 200040, China $^{1}$

M.D. Omer Cavdar Department of Cardiology, Tongji Hospital, School of Medicine, Tongji University, Shanghai 200065, China

Ph.D. Yi Shen ${ }^{2}$ Department of Geriatrics, Tongji Hospital, School of Medicine, Tongji University, Shanghai 200065, China》

1 Equal contribution as the first author

2 Corresponding author

\section{Acknowledgements}

Not applicable

\section{References}

1. Li J, Cao F, Yin HL, Huang ZJ, Lin ZT, Mao N, Sun B, Wang G. Ferroptosis: past, present and future. Cell Death Dis. $2020 ; 11(2): 88$.

2. Zhi-Zhou Shi, Ze-Wen Fan, Yun-Xia Chen, Xiu-Feng Xie, Wen Jiang, Wen-Jun Wang, Yun-Tan Qiu , Jie Bai . Ferroptosis in Carcinoma: Regulatory Mechanisms and New Method for Cancer Therapy. Onco Targets Ther.. $2019 ; 12: 11291-11304$.

3. Kang R, Kroemer G, Tang D. The Tumor Suppressor Protein p53 and the Ferroptosis Network. Free Radic Biol Med. 2019 ;133:162-168.

4. Bo Chu, Ning Kon, Delin Chen, Tongyuan Li, Tong Liu, Le Jiang, Shujuan Song, Omid Tavana, and Wei Gu.ALOX12 is required for p53-mediated tumor suppression through a distinct ferroptosis pathway. Nat Cell Biol. $2019 ; 21(5): 579-591$.

5. Miao-Qian Shi , Fei-Fei Su , Xuan Xu, Xiong-Tao Liu , Hong-Tao Wang , Wei Zhang , Xue Li , Cheng Lian, Qiang-Sun Zheng , Zhi-Chun Feng. Cilostazol suppresses angiotensin II-induced apoptosis in endothelial cells. Mol Med Rep. 2016;13(3):2597-2605.

6. R Hernanz, S Martínez-Revelles, R Palacios, A Martín,V Cachofeiro, A Aguado, L García-Redondo, M T Barrús,P R de Batista, A M Briones, M Salaices, M J Alonso.Toll-like receptor 4 contributes to vascular remodelling and endothelial dysfunction in angiotensin Il-induced hypertension. British Journal of Pharmacolog. $2015 ; 172(12): 3159-3176$.

7. Max J Männel, Carolin Fischer , Julian Thiele. A Non-Cytotoxic Resin for Micro-Stereolithography for Cell Cultures of HUVECs. Micromachines (Basel). 2020;11(3):246.

8. Chuanzhao Ji,Yang Yu,Min Zhang,Wenyan Yu, Shuo Dong. Loxoprofen Sodium Alleviates Oxidative Stress and Apoptosis Induced by Angiotensin II in Human Umbilical Vein Endothelial Cells (HUVECs). Drug Des Devel Ther. 2020;14:5087-5096. 
9. Jisu Jeong, Jiye Lee, Juyeon Lim, Soyoung Cho, Soyoung An, Myungeun Lee, Nara Yoon, Miran Seo, Soyeon Lim, Sungha Park. Soluble RAGE attenuates Angll-induced endothelial hyperpermeability by disrupting HMGB1-mediated crosstalk between AT1R and RAGE. Exp Mol Med. 2019;51(9):1-15.

10. Weifen Zhang, Chengcheng Gai, Dejun Ding, Fang Wang,Wentong Li.Targeted p53 on SmallMolecules-Induced Ferroptosis in Cancers. Front Oncol. 2018 ;8:507.

11. Gnanapradeepan K, Basu S, Barnoud T, Budina-Kolomets A, Kung CP, Murphy ME.The p53 Tumor Suppressor in the Control of Metabolism and Ferroptosis.Front Endocrinol (Lausanne). 2018 11;9:124.

12. Zhonghua Zheng, Yin Li, Gehui Jin, Tianyi Huang, Mengsha Zou, Shiwei Duan. The biological role of arachidonic acid 12-lipoxygenase (ALOX12) in various human diseases. Biomed Pharmacother. 2020 ;129:110354.

13. Dong C, Liu S, Cui Y, Guo Q.12-Lipoxygenase as a key pharmacological target in the pathogenesis of diabetic nephropathy. Eur J Pharmacol. $2020 ; 879: 173122$

14. Bo Chu, Ning Kon, Delin Chen, Tongyuan Li, Tong Liu, Le Jiang, Shujuan Song, Omid Tavana, Wei Gu .ALOX12 is required for p53-mediated tumour suppression through a distinct ferroptosis pathway. Nat. Cell Biol. 2019 ; 21(5): 579-591.

15. L Siangjong , D H Goldman, T Kriska, K M Gauthier, E M Smyth , N Puli , G Kumar , J R Falck, W B Campbell L. Vascular hepoxilin and trioxilins mediate vasorelaxation through TP receptor inhibition in mouse arteries. Acta Physiol. Acta Physiol (Oxf). 2017;219(1):188-201.

16. Hye Young Kim , Dae Won Jeong, Ho Sun Park, Tae Yoon Lee, Hee Sun Kim. Comparison of 12lipoxygenase expression in vascular smooth muscle cells from old normotensive Wistar-Kyoto rats with spontaneously hypertensive rats. Hypertens. Res. 2013;36(1): 65-73.

17. L. Thapa, C.M. He, H.P. Chen. Study on the expression of angiotensin II (ANG II) receptor subtype1(AT1R)in the placenta of pregnancy-induced hypertension. Placenta 2004;25(7):637-641.

18. Rona Limor, Marielle Kaplan, Orly Sharon, Esther Knoll, Michal Naidich, Gary Weisinger, Shlomo Keidar, Naftali Stern. Aldosterone up-regulates 12- and 15-li-poxygenase expression and LDL oxidation in human vascular smooth muscle cells,J. Cell. Biochem. J Cell Biochem. 2009;108(5): 1203-1210.

19. Maryam Farzaneh Behelgardi1, Saber Zahri1, Farhad Mashayekhi, Kamran Mansouri,S. Mohsen Asghari.A peptide mimicking the binding sites of VEGF-A and VEGF-B inhibits VEGFR-1/-2 driven angiogenesis, tumor growth and metastasis. Sci Rep. $2018 ; 8(1): 17924$.

20. Ingiridur Skirnisdottir , Tomas Seidal, Helena Åkerud. The relationship of the angiogenesis regulators VEGF-A, VEGF-R1 and VEGF-R2 to p53 status and prognostic factors in epithelial ovarian carcinoma in FIGO-stages I-II. Int J Oncol. 2016 ;48(3):998-1006.

21. Yongchuan Zhang ,Yinglin Zhang, Jin Wang, Haitao Gu. Amarogentin Inhibits Liver Cancer Cell Angiogenesis after Insufficient Radiofrequency Ablation via Affecting Stemness and the p53Dependent VEGFA/DII4/Notch1 Pathway. Biomed Res Int . 2020;2020:5391058 
22. Miles J Pfaff, Subhradip Mukhopadhyay, Mark Hoofnagle, Christine Chabasse, Rajabrata Sarkar . Sarkar. Tumor suppressor protein p53 negatively regulates ischemia-induced angiogenesis and arteriogenesis. Journal of Vascular Surgery. 2018;68(6S):222S-233S.

23. Wamidh H Talib , Lina T Al Kury. Parthenolide inhibits tumor-promoting effects of nicotine in lung cancer by inducing P53-dependent apoptosis and inhibiting VEGF expression. Biomed. Pharmacother. 2018;107:1488-1495.

24. Laura Morshaeuser, Matthias May, Maximilian Burger, Wolfgang Otto, Georg C Hutterer, Martin Pichler, Tobias Klatte, Peter Wild, Lorenz Buser, Sabine Brookman-May . p53-expression in patients with renal cell carcinoma correlates with a higher probability of disease progression and increased cancer-specific mortality after surgery but does not enhance the predictive accuracy of robust outcome models. Urol Oncol . 2018;36(3):94.e15-94.e21.

25. Xiaoxiang Chen, Kaixuan Zeng, Mu Xu1, Xiangxiang Liu, Xiuxiu Hu , Tao Xu, Bangshun He, Yuqin Pan , Huiling Sun, Shukui Wang. P53-induced miR-1249 inhibits tumor growth, metastasis, and angiogenesis by targeting VEGFA and HMGA2. Cell Death Dis. $2019 ; 10(2): 131$.

26. William S. Powella,Joshua Rokach. Biosynthesis, biological effects, and receptors of hydroxyeicosatetraenoic acids (HETEs) and oxoeicosatetraenoic acids (oxo-ETEs) derived from arachidonic acid. Biochim Biophys Acta. 2015; 1851(4): 340-355.

\section{Tables}

Tab 1 Effects of different concentrations of Angll on MAD, GSH, ALOX12, VEGFA and P53

$\begin{array}{lllllllll}\text { AngII (uM) } & 0 & 0.1 & 1.0 & 10 & 100 & 1000 & F & p\end{array}$

VEGFA $\quad 1 \pm 0.235 \quad 0.81 \pm 0.07 \quad 0.72 \pm 0.026 \quad 0.623 \pm 0.05 \quad 0.223 \pm 0.06 \quad 0.293 \pm 0.075 \quad 75.816 \quad 0.000$

ALOX12 $0.1 \pm 0.01903 \quad 0.1 \pm 0.02 \quad 0.103 \pm 0.022 \quad 0.101 \pm 0.033 \quad 0.754 \pm 0.015 \quad 0.677 \pm 0.017 \quad 30.396 \quad 0.000$

P53 $\quad 0.1 \pm 0.114 \quad 0.113 \pm 0.042 \quad 0.122 \pm 0.014 \quad 0.285 \pm 0.015 \quad 0.415 \pm 0.082 \quad 0.374 \pm 0.014 \quad 75.117 \quad 0.000$

MDA $\quad 0.297 \pm 0.067 \quad 0.305 \pm 0.047 \quad 0.345 \pm 0.091 \quad 0.412 \pm 0.075 \quad 0.987 \pm 0.015 \quad 1.01 \pm 0.079 \quad 75.548 \quad 0.000$

GSH $\quad 2.969 \pm 0.441 \quad 3.03 \pm 0.354 \quad 3.275 \pm 0.299 \quad 3.137 \pm 0.297 \quad 8.7 \pm 0.16 \quad 8.9 \pm 0.129 \quad 25.399 \quad 0.000$
F $\quad 3.123$
$P \quad 0.049$ 
Tab 2 Multiple regression analysis to investigate the relationship of Ang II and ALOX12, P53, VEGFA,MDA,GSH in vascular endothelial cells

\begin{tabular}{lccccc}
\hline ALOX12 & P53 & VEGFA & MDA & GSH \\
& & & & & \\
& & & & & \\
Standardized $\beta$ & 0.696 & 0.364 & -0.71 & 0.98 & 0.33 \\
p value & 0.002 & 0.045 & 0.008 & 0.001 & 0.036 \\
\hline
\end{tabular}

Tab 3 Effects of Losartan, Pofithrin-X on MAD, GSH, ALOX12, VEGFA and P53

\begin{tabular}{lccccc}
\hline & P21 & VEGFA & ALOX12 & MDA & GSH \\
\hline & & & & & \\
Ang II & $0.813 \pm 0.057$ & $0.492 \pm 0.019$ & $0.938 \pm 0.051$ & $0.987 \pm 0.015$ & $8.7 \pm 0.16$ \\
& & & & & \\
AngII+Pof ithrin- 8 & $0.788 \pm 0.033$ & $0.501 \pm 0.021$ & $0.445 \pm 0.019$ & $0.56 \pm 0.012$ & $4.158 \pm 0.091$ \\
& & & & & \\
AngII+Losartan & $0.868 \pm 0.025$ & $0.595 \pm 0.031$ & $0.52 \pm 0.02$ & $0.714 \pm 0.08$ & $5.475 \pm 0.052$ \\
& & & & & \\
AngII+Losartan+Pofithrin-X & $0.637 \pm 0.048$ & $0.602 \pm 0.049$ & $0.297 \pm 0.028$ & $0.394 \pm 0.022$ & $2.236 \pm 0.135$ \\
& & & & & \\
F & 32.37 & 18.112 & 11.911 & 43.09 & 52.667 \\
P & 0.000 & 0.000 & 0.000 & 0.000 & 0.000 \\
\hline
\end{tabular}

\section{Figures}



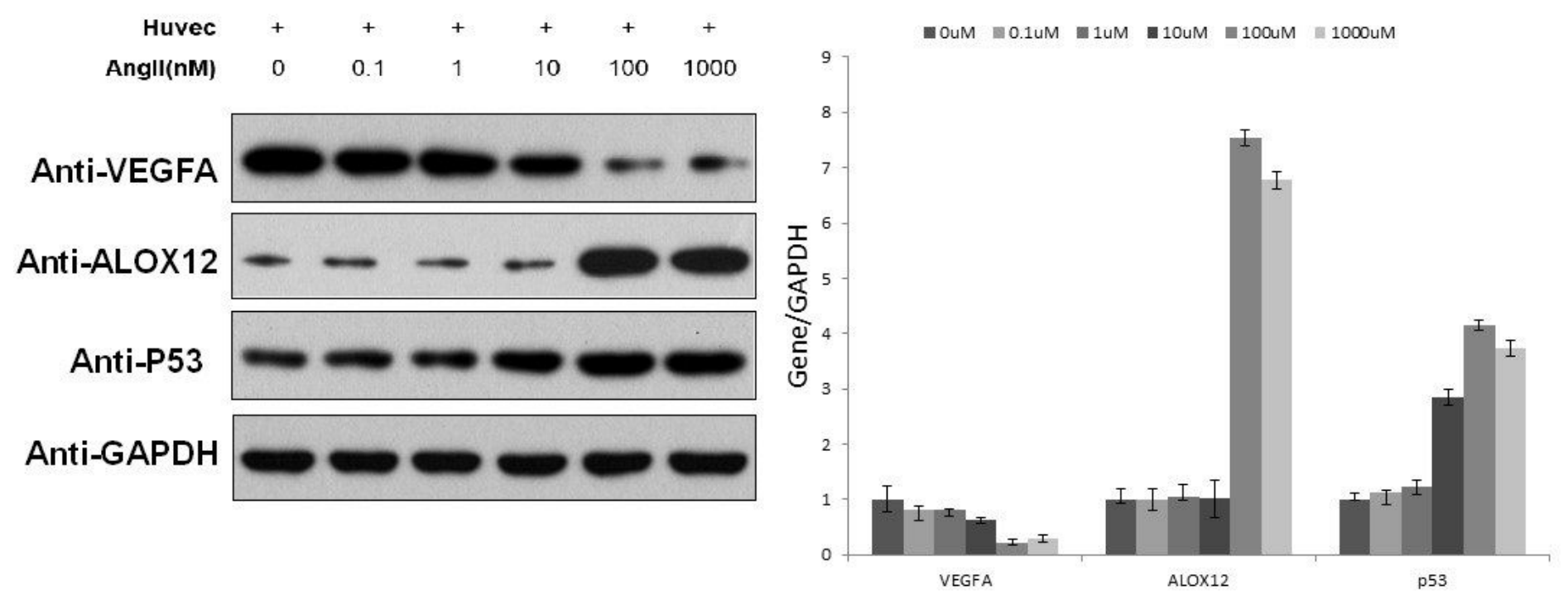

a
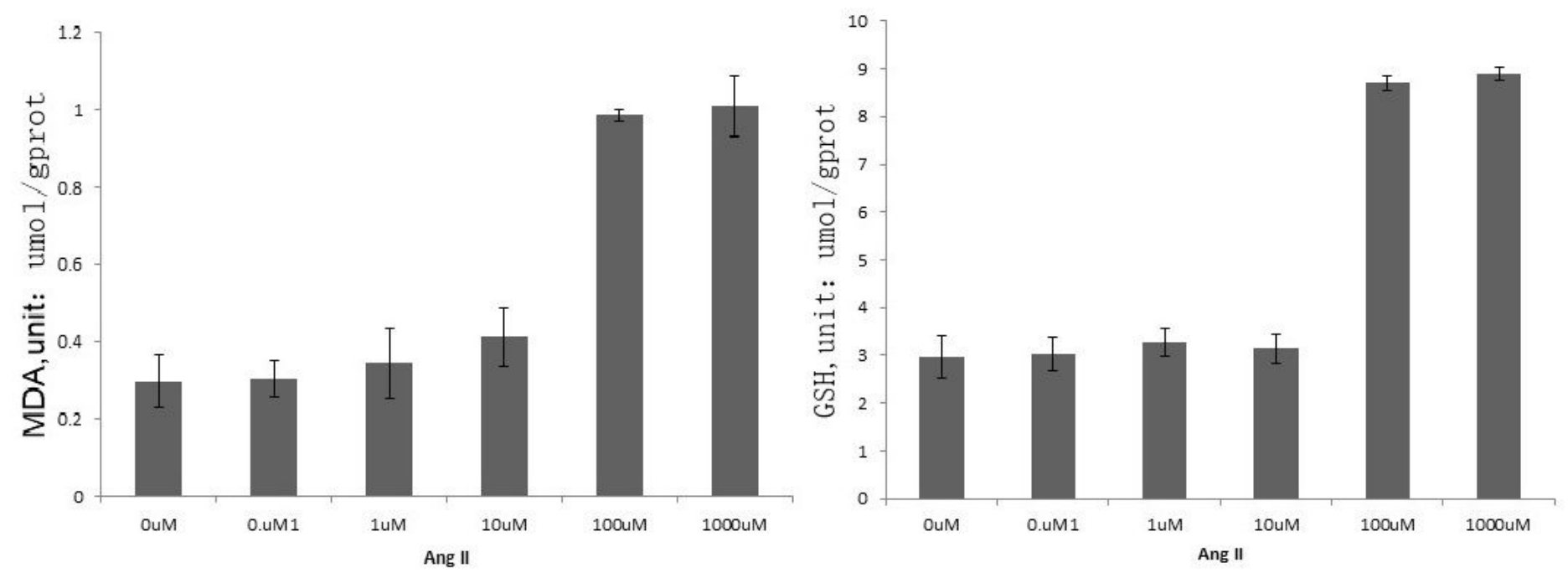

b

\section{Figure 1}

The relation between vascular endothelial ferroptosis and dose of Ang II. All P values were calculated using Student's t-test and one-way analysis of variance (ANOVA). Detailed statistical tests are described in the Methods. Raw data are provided in Table 1, 2. a. Western blot analysis of ALOX12『VEGFA匹P53 in HUVECs to be treated with different dose of Ang II for 48hours. The experiments were repeated twice, independently, with similar results. Error bars are mean \pm s.d., $n=3$ independent experiments. b. The level of GSH and MDA was measured by GSH and MDA quantification kit in in HUVECs with different dose of Ang II treatment. Error bars are mean \pm s.d., $n=3$ independent experiments. 

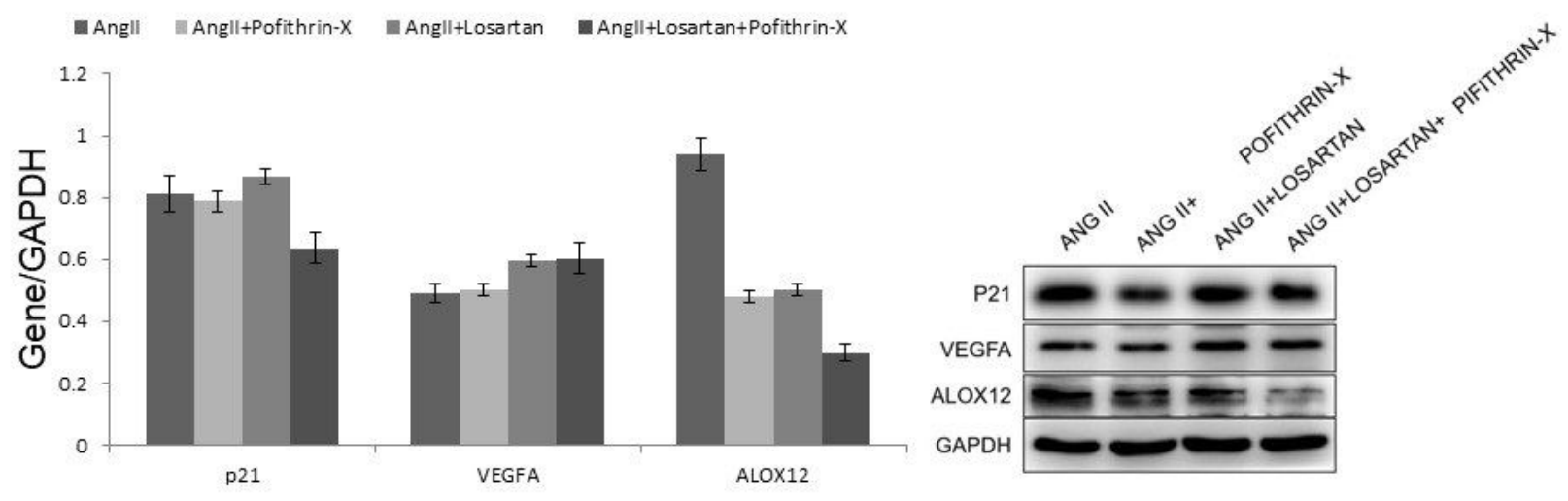

a
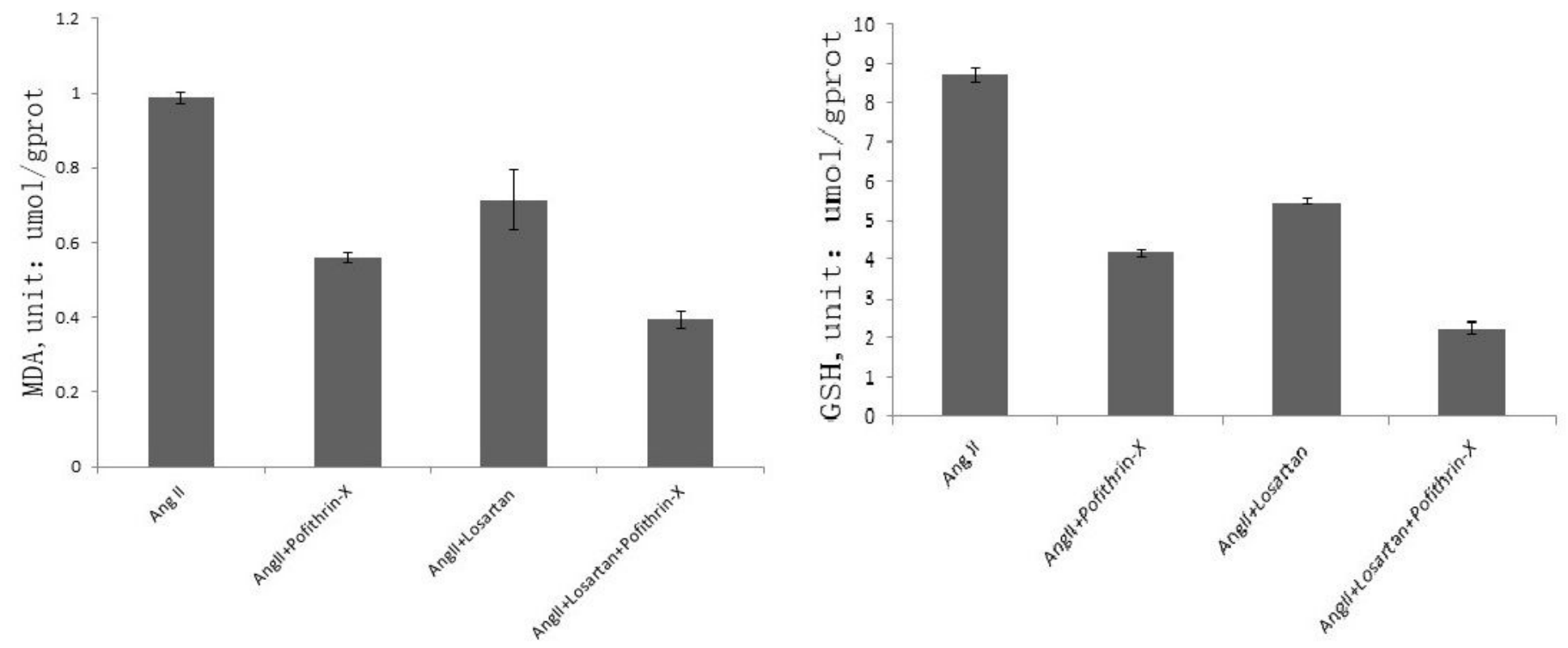

b

Figure 2

Losartan or/and Pofithrin-X prevented Ang-II- induced reduction of MDA, GSH, P21, ALOX12 and an increase in expression of VEGFA. All $P$ values were calculated using Student's t-test and one-way analysis of variance (ANOVA). Detailed statistical tests are described in the Methods. Raw data are provided in Table 3. a. Western blot analysis of HUVECs pre-incubated with Angll囚then treated with Losartan or/and Pofithrin- $X$ as indicated for $48 \mathrm{~h}$. The experiments were repeated twice, independently, with similar results. Error bars are mean \pm s.d., $n=3$ independent experiments. b. Effect of Losartan or/and Pofithrin-X on MAD and GSH in HUVECs. The level of GSH and MDA was measured by GSH and MDA quantification kit in HUVECs pre-incubated with Angll囚then treated with Losartan or/and Pofithrin-X as indicated for 48h. Error bars are mean $\pm s . d ., n=3$ independent experiments. 